\title{
On the performance of code word diversity based quasi orthogonal space time block codes in multiple-input- multiple-output systems
}

\author{
Senthilkumar Kumaraswamy ${ }^{1}$, Palanivelan Manickavelu², Noormohammed Valimohamad ${ }^{3}$, \\ Helanvidhya Thankaraj ${ }^{4}$, Yogalakshmi Venkatesan ${ }^{5}$, Bakyalakshmi Veeraragavan ${ }^{6}$ \\ 1,2,4,5,6 Department of Electronics and Communication Engineering, Rajalakshmi Engineering College, Chennai, India \\ ${ }^{3}$ School of Electronics Engineering, Vellore Institute of Technology, Vellore, TN, India
}

\begin{tabular}{l} 
Article Info \\
\hline Article history: \\
Received Jun 12, 2019 \\
Revised Nov 13, 2019 \\
Accepted Nov 22, 2019 \\
\hline
\end{tabular}

\section{Keywords:}

Bit error rate

Diversity

Multiple-input-multiple-output

Quasi orthogonal space time

block code

\begin{abstract}
In the recent past, a lot of researches have been put into designing a MultipleInput-Multiple-Output (MIMO) system to provide multimedia services with higher quality and at higher data rate. On par with these requirements, a novel Quasi Orthogonal Space Time Block Code (QOSTBC) scheme based on code word diversity is proposed, which is a multi-dimensional approach, in this paper. The term code word diversity is coined, since the information symbols were spread across many code words in addition to traditional time and spatial spreading, without increasing transmission power and bandwidth. The receiver with perfect channel state information estimates the transmitted symbols with less probability of error, as more number of samples is available to estimate given number of symbols due to the extra diversity due to code words. The simulation results show a significant improvement in the Bit Error Rate (BER) performance of the proposed scheme when compared with the conventional schemes.
\end{abstract}

Copyright $(2020$ Institute of Advanced Engineering and Science. All rights reserved.

\section{Corresponding Author:}

Senthilkumar Kumaraswamy,

Department of Electronics and Communication Engineering,

Rajalakshmi Engineering College,

Rajalakshmi Nagar, Thandalam, Chennai, Tamil Nadu 602105, India.

Email: senthilkumar.kumaraswamy@rajalakshmi.edu.in

\section{INTRODUCTION}

Higher data rate and reliability are the demands of the modern day applications and are met by Multiple-Input-Multiple-Output (MIMO) technique. Exhaustive research was carried out in the past and still it is a field of interest. MIMO systems have become the field of attraction in recent times, as they support high speed applications without demanding extra communication resources like power and bandwidth [1, 2]. Space-Time Block Coding (STBC) is a scheme developed for MIMO systems to provide higher diversity gain and coding gain [3]. The subcarrier power allocation algorithms to meet the requirements of next generation wireless technology are proposed in [4-6]. In [7], a multipath diversity scheme in conjunction with OFDM is proposed to improve the BER performance of a MIMO system. Orthogonal Space Time Block Code (OSTBC) is a kind of STBC, having faster Maximum Likelihood (ML) decoding ability due to its orthogonal property [8], however, suffers a drawback of ability to offer full rate only for two antennas, for complex constellations [9] and [10]. QOSTBC has gained the attention as an alternative to overcome this drawback with a compromise in orthogonality, which in turn compromises with diversity order and decoding complexity [11]. To improve Bit Error Rate (BER) performance of QOSTBC, optimum constellation rotation is proposed in [12]. In [13], a receive antenna selection scheme without feedback requirement for exploiting the advantages of MIMO is proposed. Cooperative diversity schemes for exploiting advantages of MIMO are proposed in [14-20]. In contrast to the open loop QOSTBCs mentioned above, several closed loop QOSTBC schemes 
are presented in [21-24] for better performance. The drawbacks of these schemes are higher cost and complexity due to the feedback. In $[25,26]$, the channel state information is computed at the receiver and fed back to the transmitter via single bit of information. Based on the feedback, the transmitter selects one of its two code words for transmission, which suits better for the present channel conditions. In [27], a scheme that selects best code word for a given channel condition is presented. All these works are based on the truth that the channels respond differently for different spatial locations (i.e. spatial diversity), at different instances of time (i.e. temporal diversity), for different frequencies (i.e. frequency diversity) and for different polarizations (i.e. polarization diversity).

Similarly, the channels respond differently for different QOSTBC code words, for one code word, the performance may be good at one instant of time and the same may yield a poor performance later. A multidimensional approach for space-time coding is proposed in [28], which combined spatial and temporal diversity with spatial multiplexing. The three dimensional tensor coding considered transmitting antenna, time and data stream as its dimensions. In [29], the authors have proposed Multiple Khatri-Rao Space-Time (MKRST) code for improving the performance of MIMO systems by providing additional diversity either in time or space. In [30], the authors have proposed Double Orthogonal Vandermonde constrained Alternating Least Square (DOVALS) algorithm to utilize the orthogonal structures in the factored matrices. From the literature, it is understood that increasing the number of received signals without increasing the frequency, time and power requirement is still an open problem. It is also clear that the full potential of diversity is not fully exploited. The idea of the proposed work is that all the code words that are transmitted will not suffer the same channel impairments and with a hope that at least one will be received in good condition, similar to the concepts of time, space and frequency diversity. But, the proposed work makes use of code word diversity, in addition to space and time diversity for improving the BER performance of the system. To the best of our knowledge, this is the first work on multidimensional approach for code word diversity with QOSTBC. The proposed work makes use of code word diversity in addition to the space and time diversity. The proposed work transmits multiple code words simultaneously with the same power and bandwidth, without requiring feedback. Thus, the performance of the proposed work is better than the conventional schemes, thanks to the extra code word diversity.

The main contribution of the proposed scheme is the increase in the number of received signals by increasing the number of code words for a fixed number of symbols to be estimated. The following are the notations used in this paper. The superscripts $(.)^{*},(.)^{T}$ and $(.)^{H}$ denotes complex conjugate, transpose and transconjugate (i.e. Hermitian transpose) respectively. Extraction of diagonal elements from a matrix is denoted by diag (.). The Moore Penrose pseudo inverse of a matrix and Frobenius norm are denoted by (.) $)^{\dagger}$ and $\|\cdot\|_{F}$ respectively. The rest of the paper is organized as follows. System model is described in section 2. Performance analysis is presented in section 3. Results and discussions are presented in section 4. Finally, section 5 concludes the paper.

\section{SYSTEM MODEL}

We have considered quasi-static, flat fading Rayleigh channel which remains constant during transmission of one block and changes for next. The transmitter of the proposed system consists of a Code word-Space-Time (CST) encoder, which spreads the complex information symbols from the symbol mapper, across three dimensions i.e. in space, time and code words. There are $A$ transmitting antennas at the transmitter, which simultaneously transmits $C$ QOSTBC code words in $N$ time slots. The receiver consists of $K$ antennas, which adds one more dimension and hence the received signal is a four dimensional signal. With the perfect channel state information at the receiver, the Code word-Space-Time decoder estimates the transmitted symbols. The block diagram of the transmitter and receiver of proposed code word diversity based QOSTBC scheme is given in Figure 1 and Figure 2.

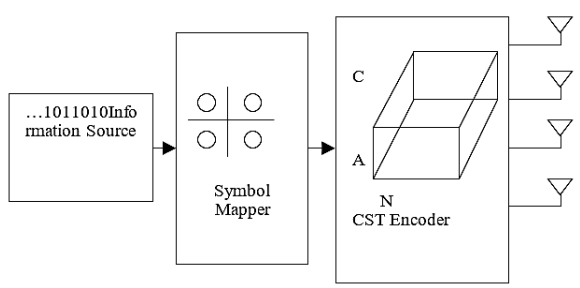

Figure 1. Transmitter of the proposed code word diversity based QOSTBC scheme 


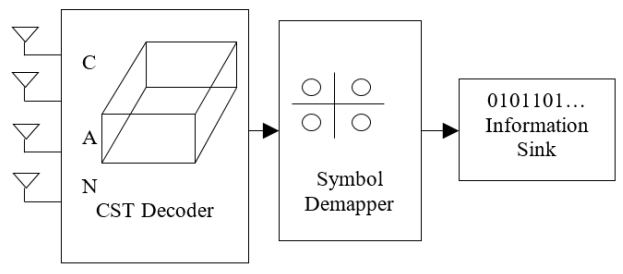

Figure 2. Receiver of the proposed code word diversity based QOSTBC scheme

The incoming bit stream (i.e. $2 N$ bits per block) is mapped onto a complex constellation like QAM or QPSK and a symbol sequence $S$ of length $N$ is obtained and is given by (1) as

$$
S=\left[s_{1}, s_{2}, \ldots, s_{N}\right] \in \mathcal{C}^{1 \times N}
$$

These information symbols are spread across time, space and code words (3-D) by multiplying with a 3dimensional information spreading matrix $W$ and is given by (2) as

$$
W=e^{j 2 \pi a n c \mid A N C} \in \mathcal{C}^{A \times N \times C}
$$

where $A$ is the number of transmitting antennas, $N$ is the number of time slots required for transmitting a code word, $C$ is the number of code words that are simultaneously transmitted. The information spread matrix $G$ is obtained by element wise product of $W$ in (2) and $S$ in (1) along the dimension $N$ and is given by (3)

$$
G_{A C}^{N}=W_{A C}^{N} S^{A \times N \times C}
$$

The information spread matrix $G$ is transmitted through $A$ transmit antennas in $N$ successive time slots. The transmitted signal $X$ is obtained by element wise product of $G$ and $H$ along the dimension $N$ and is given by (4) as

$$
\mathrm{X}_{\mathrm{AC}}^{\mathrm{N}}=\mathrm{G}_{\mathrm{AC}}^{\mathrm{N}} \mathrm{H}_{\mathrm{ACK}}^{\mathrm{N}}
$$

where $H \in \mathcal{C}^{A \times N \times C \times K}$ is the channel matrix whose entries are independent and identically distributed with zero mean and unit variance. The received signal $R$ is given by (5) as

$$
\mathrm{R}=\mathrm{X}+\mathrm{Z} \in \mathcal{C}^{\mathrm{A} \times \mathrm{N} \times \mathrm{C} \times \mathrm{K}}
$$

where the noise $Z \in \mathcal{C}^{A \times N \times C \times K}$, whose entries are independent samples of a circularly symmetric complex Gaussian random variable with zero mean and unit variance. The symbols are decoded at the receiver by making use of matrix unfolding as follows. The unfolding is done across the plane $C$. For better understanding about the unfolding of a matrix, [25] can be referred. The unfolded received signal $R_{\text {unfolded }}$ and the unfolded spreading matrix is $W_{\text {unfolded }}$ given by (6) as

$$
\begin{aligned}
\mathrm{R}_{\text {unfolded }} & =\left[\begin{array}{c}
\mathrm{R}_{. .1} \\
\vdots \\
\mathrm{R}_{. \mathrm{c}}
\end{array}\right] \text { and } \\
\mathrm{W}_{\text {unfolded }} & =\left[\begin{array}{c}
\mathrm{W}_{.1} \\
\vdots \\
\mathrm{W}_{. \mathrm{c}}
\end{array}\right] \in \mathcal{C}^{\mathrm{ACK} \times \mathrm{N}}
\end{aligned}
$$

The unfolded channel $H_{\text {unfolded }}$ matrix is given by (7) as

$$
R_{\text {unfolded }}=\left[\begin{array}{c}
H_{.1} \\
\vdots \\
H_{. . c}
\end{array}\right] \in \mathcal{C} A C K \times N
$$

The unfolded received signal $X_{\text {unfolded }}$ is represented by (8) as

$$
X_{\text {unfolded }}=G_{\text {unfolded }} * H_{\text {unfolded }}
$$




$$
\begin{aligned}
& =\left[W_{\text {unfolded }} * S\right] * H_{\text {unfolded }} \\
& =\left[W_{\text {unfolded }} * H_{\text {unfolded }}\right] * S
\end{aligned}
$$

The transmitted symbols $\hat{S}$ can be estimated at the receiver using (10) and it is given by (11) as

$$
\hat{S}=\operatorname{diag}\left[\left[W_{\text {unfolded }} * H_{\text {unfolded }}\right]^{\dagger} \cdot * X_{\text {unfolded }}\right]
$$

From (11), it can be understood that a blind joint symbol and channel estimation is possible using Alternate Least Square (ALS) technique.

\section{PERFORMANCE ANALYSIS}

In this section, we have analyzed the performance of the proposed scheme and presented the equation for maximum diversity gain. In general, the Pair wise Error Probability (PEP) of the Maximum Likelihood (ML) estimator of the code word matrix is used as a measure of performance. The equation for the maximum diversity gain can be derived from PEP. The PEP of the ML estimator is as follows.

$$
P(S \rightarrow \hat{S} \mid H)=Q\left(\|X-X\|_{F} / 2 \sqrt{N_{o} / 2}\right)
$$

Due to space constraints, we skip the intermediate steps and present the final result of the derivation for maximum diversity gain $d_{\max }$ in (13) as

$$
d_{\max }=A \times C \times K \times N
$$

The performance comparison between the conventional schemes and the proposed scheme is presented in Table 1.

Table 1 Comparison of diversity gain and code rate of the proposed code word diversity based QOSTBC with conventional schemes

\begin{tabular}{lll}
\hline Scheme & Diversity gain & Code rate \\
\hline Zhu Chen code [25] & Full diversity & Full rate \\
& $(M \times N)$ & (Unity) \\
Gerard code [28] & Full diversity & $<$ Full rate \\
& $(K \times P \times J \times \min (M, R))$ & $\left(\frac{P}{R}\right)$ \\
Proposed code word diversity & Full diversity & Full rate \\
& $(A \times C \times K \times N)$ & (Unity) \\
\hline
\end{tabular}

\section{RESULTS AND DISCUSSIONS}

In this section, we have evaluated the error performance of the proposed code word diversity scheme in a quasi static flat fading channel by means of Monte Carlo simulations. The receiver is assumed to have perfect channel state information. The simulation parameters are shown in Table 2. Figure 3 compares the proposed code word diversity scheme with conventional unrotated QOSTBC, rotated QOSTBC, Zhu Chen code [25], Gerard code [28], MKRST [29] and DOVALS [30]. We adopt QPSK constellation for simulating the BER over SNR. The BER for paper [21] is evaluated for a block length of four $(P=4)$, spreading length of one $(J=1)$, with two transmitting $(M=2)$ and two receiving antennas $(K=2)$. For simulating paper [25], unrotated QOSTBC and rotated QOSTBC, we have considered four transmitting antennas and one receiving antenna as mentioned in the original papers. For simulating the proposed code word diversity scheme, we have assumed four transmitting antennas $(A=4)$ for transmitting three code words $(C=3)$ simultaneously and one receiving antenna $(K=1)$. From the Figure 3, it is observed that BER of all the schemes decreases with increase in SNR. It is also clear that the proposed scheme outperforms the schemes of [25] and [28], due to spreading across code words in addition to time and space. The proposed scheme achieves a SNR gain of $6.7 \mathrm{~dB}$ at the BER of $10^{-5}$ over [25] and around $2 \mathrm{~dB}$ over [28]. The proposed scheme achieves a SNR gain of $2 \mathrm{~dB}$ at the BER of $10^{-5}$ over [29] and around $1.4 \mathrm{~dB}$ over [30]. 
In Figure 4, we show the effect of number of code words on the BER performance of the proposed code word diversity scheme. From the figure, it is observed that BER of all the schemes decreases with increase in SNR. For a given number of receive antenna, the BER decreases with increase in number of code words. Also, for a given number of code words, the BER decreases with increase in number of receive antenna. We found that the proposed scheme with $C=3, K=3$ outperforms proposed scheme with other values of $C$ and $K$, due to increase in number of samples for estimating the same number of symbols, but at the cost of increase in computational complexity. The effect of constellation size on the BER performance of the proposed scheme is investigated for various values of $M$, with single receive antenna.

Table 2. Simulation parameters

\begin{tabular}{ll}
\hline Parameter & Value \\
\hline Modulation & QAM and QPSK \\
Noise & AWGN \\
Channel & quasi static flat fading \\
Number of receive antennas & $1,2,3$ and 4 \\
Number of receive antennas & $1,2,3$ and 4 \\
\hline
\end{tabular}

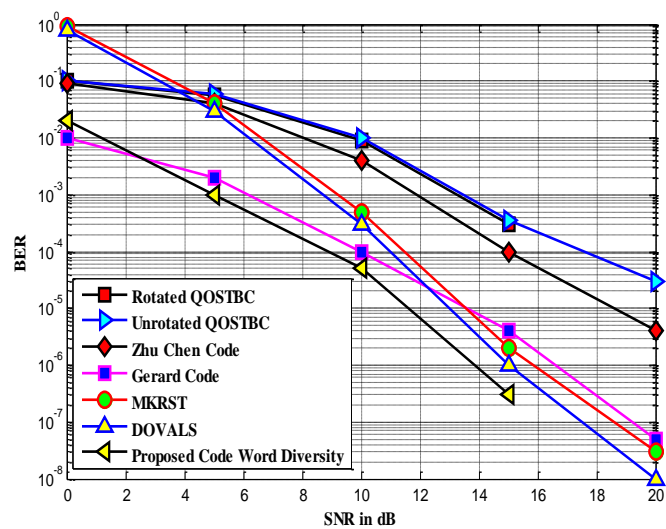

Figure 3. Comparison of BER performance of proposed code word diversity based QOSTBC with conventional schemes

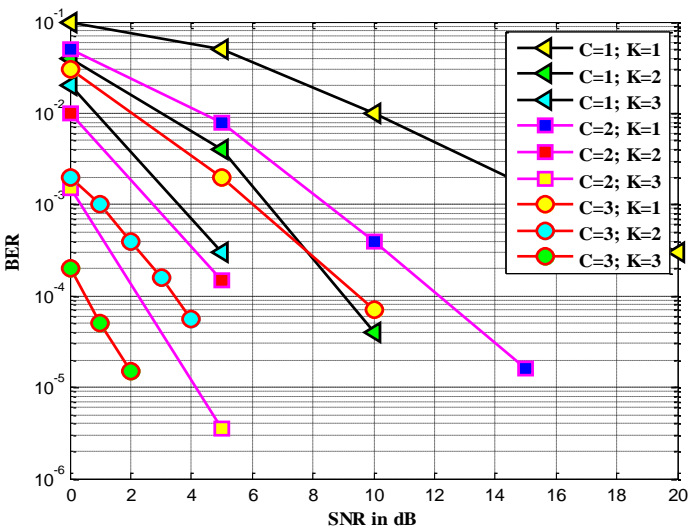

Figure 4. Influence of the number of code words $C$ and number of receive antennas $\mathrm{K}$ on the BER performance of the proposed code word diversity based QOSTBC

Figure 5 depicts the influence of 128-QAM on BER with $C=1,2$ and 3. It is observed that the BER performance of the proposed scheme with 128-QAM improves with increase in SNR for all the $C$ values considered. At a SNR of $10 \mathrm{~dB}$, the BER values for $C=1,2$ and 3 are $9 \times 10^{-2}, 4 \times 10^{-2}$ and $2.8 \times 10^{-2}$ respectively. From the figure, it is evident that the BER performance of a MIMO system improves with code word diversity (i.e. $C=2$ and $C=3$ ) when compared to the scheme without code word diversity (i.e. $C=1$ ).

Figure 6 shows the impact of 64-QAM on BER with $C=1,2$ and 3. It is observed that the BER performance of the proposed scheme with 64-QAM improves with increase in SNR for all the $C$ values considered. At a SNR of $10 \mathrm{~dB}$, the BER values for $C=1,2$ and 3 are $7 \times 10^{-2}, 2.8 \times 10^{-2}$ and $2 \times 10^{-2}$ respectively. From the figure, it is evident that the BER performance of a MIMO system improves with code word diversity (i.e. $C=2$ and $C=3$ ) when compared to the scheme without code word diversity (i.e. $C=1$ ). On comparing Figure 5 and Figure 6, it is observed that the BER for $C=3$ with 128 -QAM is $2.8 \times 10^{-2}$ at $10 \mathrm{~dB}$, whereas it is $2 \times 10^{-2}$ for $C=3$ with 64-QAM. Therefore, it is clear that the BER performance of the proposed scheme improves with reduction in constellation size.

Figure 7 illustrates the effect of 16-QAM on BER with $C=1,2$ and 3. It is observed that the BER performance of the proposed scheme with 16-QAM improves with increase in SNR for all the $C$ values considered. At a SNR of $10 \mathrm{~dB}$, the BER values for $C=1,2$ and 3 are $5 \times 10^{-2}, 1 \times 10^{-2}$ and $3.5 \times 10^{-3}$ respectively. From the figure, it is evident that the BER performance of a MIMO system improves with code word diversity (i.e. $C=2$ and $C=3$ ) when compared to the scheme without code word diversity (i.e. $C=1$ ). On comparing Figure 6 and Figure 7, it is observed that the BER for $C=3$ with 64-QAM is $20 \times 10^{-3}$ at $10 \mathrm{~dB}$, whereas it is $3.5 \times 10^{-3}$ for $C=3$ with 16 -QAM. Therefore, it is clear that the BER performance of the proposed scheme improves with reduction in constellation size. 
Figure 8 depicts the influence of 4-QAM on BER with $C=1,2$ and 3. It is observed that the BER performance of the proposed scheme with 4-QAM improves with increase in SNR for all the $C$ values considered. At a SNR of $10 \mathrm{~dB}$, the BER values for $C=1,2$ and 3 are $1 \times 10^{-2}, 2.5 \times 10^{-4}$ and $4 \times 10^{-5}$ respectively. From the figure, it is evident that the BER performance of a MIMO system improves with code word diversity (i.e. $C=2$ and $C=3$ ) when compared to the scheme without code word diversity (i.e. $C=1$ ). On comparing Figure 7 and Figure 8, it is observed that the BER for $C=3$ with $4-\mathrm{QAM}$ is $3.5 \times 10^{-5}$ at $10 \mathrm{~dB}$, whereas it is $4 \times 10^{-}$ ${ }^{5}$ for $C=3$ with 16-QAM. Therefore, it is clear that the BER performance of the proposed scheme improves with reduction in constellation size. From the figures, it is clear that the BER performance of the proposed scheme degrades with increase in constellation size for given number of code words. Also it is observed that the BER performance improves with increase in number of code words for a given constellation size. From the figures it is clear that larger number of code words with smaller constellation size would be beneficial.

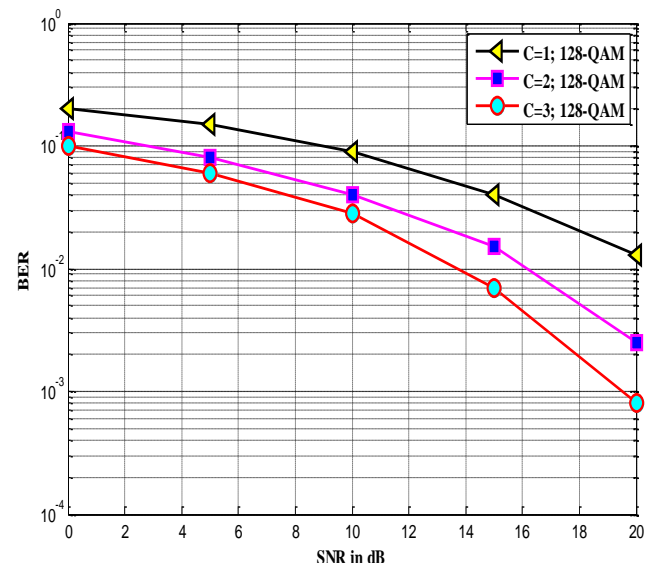

Figure 5. Influence of 128-QAM on the BER performance of the proposed code word diversity based QOSTBC for various values of C

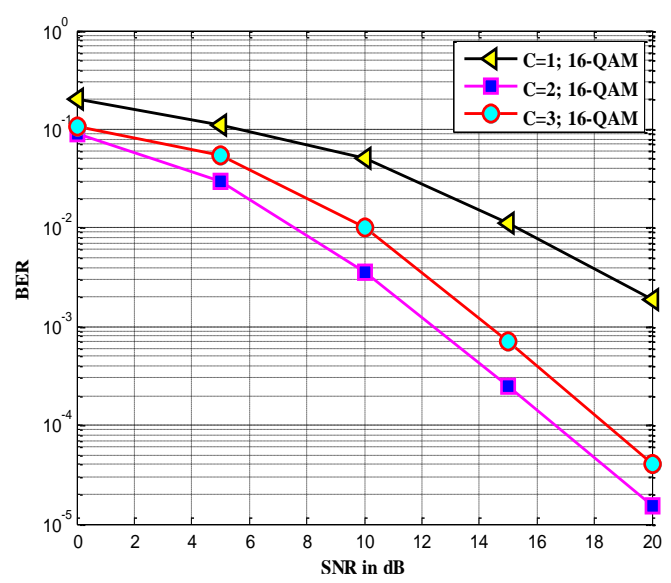

Figure 7. Influence of 16-QAM on the BER performance of the proposed code word diversity based QOSTBC for various values of $C$

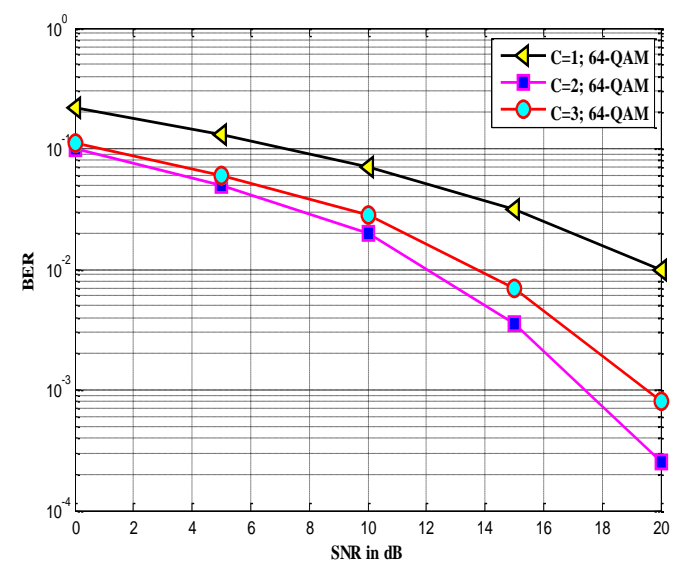

Figure 6. Influence of 64-QAM on the BER performance of the proposed code word diversity based QOSTBC for various values of $C$

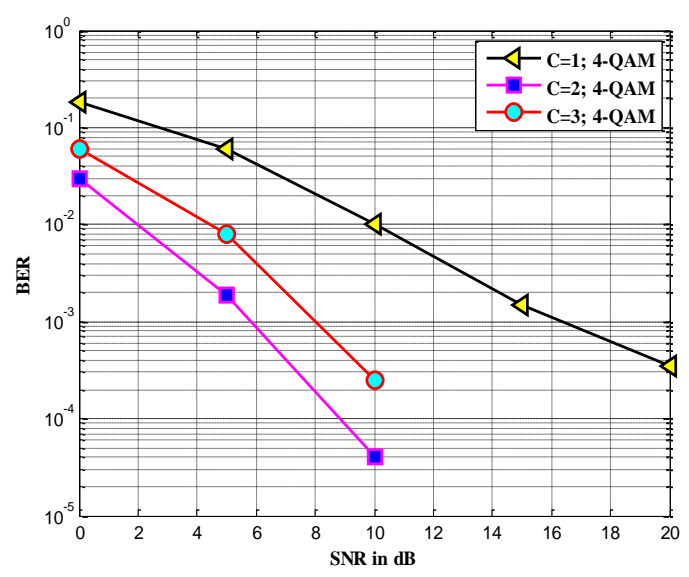

Figure 8 Influence of 4-QAM on the BER performance of the proposed codeword diversity based QOSTBC for various values of $C$

\section{CONCLUSION}

We have presented a novel code word diversity scheme for QOSTBC in this paper. From the simulated results, it is evident that the addition of code word diversity to the conventional space and time diversity, achieves a SNR gain of $6.7 \mathrm{~dB}$ at the BER of $10^{-5}$ over Zhu Chen code and around $2 \mathrm{~dB}$ over Gerard code and MKRST scheme, and around $1.4 \mathrm{~dB}$ over DOVALS scheme without increasing transmission power and bandwidth. The BER performance of the proposed code improves with increase in the number of code words, 
across which the information symbols are spread, at the cost of increase in computational complexity. In future, the proposed work can be extended by considering frequency diversity as quasi orthogonal space-timefrequency code along with code word diversity. Also the performance of the proposed work can be improved by incorporating error correcting codes such as turbo and low density parity check codes.

\section{REFERENCES}

[1] G. J. Foschini, and M. J. Gans, "On limits of wireless communications in a fading environment when using multiple antennas," Wireless Personal Communications, vol. 6, no. 3, pp. 311-335, 1998.

[2] S. M. Alamouti, "A simple transmit diversity technique for wireless communications," IEEE Journal on Selected Areas in Communications, vol. 16, no. 8, pp. 1451-1458, 1998.

[3] B. Hassibi, and B. M. Hochwald, "High-rate codes that are linear in space and time," IEEE transactions on Information Theory, vol. 48, no. 7, pp.1804-1824, 2002.

[4] S. Chitra, and N. Kumaratharan, "Multimedia transmission in MC-CDMA using adaptive subcarrier power allocation and CFO compensation," International Journal of Electronics, vol. 105, no. 2, pp. 289-302, Oct. 2017.

[5] T. Ilavarasan, S. Brindha, and M. Meenakshi, "Improved fibre nonlinearity in dispersion managed optical OFDM links,” Optics Communications, vol. 385, pp. 87-91, Oct. 2016.

[6] M. Palanivelan, and K. Senthil Kumar, "An OGA based approach for PAPR reduction in OFDM systems," International Conference on Wireless Communication and Sensor Computing (ICWCSC 2010), India, Jan. 2010.

[7] Hussein A. Leftah, and Huda N. Alminshid, "Channel capacity and performance evaluation of precoded MIMOOFDM system with large-size constellation," International Journal of Electrical and Computer Engineering, vol. 9, no. 6 , pp. 5024-5030, Dec. 2019.

[8] Hamid Jafarkhani, Space-Time Coding Theory and Practice, Cambridge University Press, New York, 2005.

[9] K. Senthilkumar, and R. Amutha, "An algorithm for energy efficient cooperative communication in wireless sensor networks," KSII Transactions on Internet and Information Systems, vol. 10, no. 7, pp. 3080-3099, Jul. 2006.

[10] K. Senthilkumar, and R. Amutha, "Energy efficient wireless body area network using receive diversity," Journal of Engineering Science and Technology, vol. 13, no. 8, pp. 2616-2631, Aug. 2018.

[11] K. Senthilkumar, and R. Amutha, "Energy efficient cooperative communication using QOSTBC in wireless sensor networks," International Journal of Advanced Engineering and Technology, vol. 7, no. 1, pp. 244-251, Mar. 2016.

[12] W. Su, and X. G. Xia, "Signal constellations for quasi-orthogonal space-time block codes with full diversity," IEEE Transactions on Information Theory, vol. 50, no. 10, pp. 2331-2347, Oct. 2004.

[13] J. K. Milleth, K. Giridhar, and D. Jalihal, "On channel orthogonalization using space-time bock coding with partial feedback,” IEEE Transactions on communication, vol. 54, no. 6, pp. 1121-1130, Jun. 2006.

[14] Anuj Singal, and Deepak Kedia, "Performance Analysis of Antenna Selection Techniques in MIMO-OFDM System with Hardware Impairments: Energy Efficiency perspective," International Journal of Electrical and Computer Engineering, vol. 8, no. 4, pp. 2272-2279, Aug. 2018.

[15] K Senthil Kumar, R. Amutha, and TLK Snehapiriya, "Energy Efficient V-MIMO using Turbo Codes in Wireless Sensor Networks," Second IEEE International Conference on Computing and Communication Technologies (ICCCT'17), India, Apr. 2017.

[16] K Senthil Kumar, R. Amutha, and R. Priyanga, "MPSK modulation based energy efficient wireless body area network," International Journal of Advanced Research Trends in Engineering and Technology, vol. 4, no. 19, pp. 272-276, Apr 2017.

[17] Qian He, Zhen Wang, Jianbin Hu and Rick S. Blum, "Performance gains from cooperative MIMO radar and MIMO communication systems," IEEE Signal Processing Letters, vol. 26, no. 1, pp. 194-198, 2018.

[18] K. Senthilkumar, and R. Amutha, "Energy Efficient Cooperative Communication in Wireless Sensor Network using turbo codes," Australian Journal of Electrical and Electronics Engineering, vol. 12, no. 4, pp. 293-300, Nov. 2015.

[19] Jaipreet Kaur, and Maninder Lal Singh, "User assisted cooperative relaying in beamspace massive MIMO NOMA based systems for millimeter wave communications," China Communications, vol. 16, no. 6, pp. 103-113, Jul. 2019.

[20] K. Senthilkumar, and R. Amutha, "Extended Lifetime with Minimum End-to-End Energy Consumption Scheme for Virtual Multiple-Input-Multiple-Output in Wireless Sensor Networks," Australian Journal of Electrical and Electronics Engineering, vol. 16, no. 4, pp. 1-12, Oct. 2019.

[21] K. Senthil Kumar, M. Kanthimathi, and R. Amutha, "Energy efficiency analysis of differential cooperative algorithm in wireless sensor network," Cluster Computing, pp. 1-9, Jan. 2018.

[22] M. Kanthimathi, R. Amutha, and K. Senthil Kumar, "Energy efficient differential cooperative MIMO algorithm for wireless sensor network," Wireless Personal Communications, vol. 103, no. 4, pp. 2715-2728, Dec. 2018.

[23] J. Akhtar, and D. Gesbert, "Extending orthogonal block codes with partial feedback," IEEE Transactions on wireless communications, vol. 3, no. 6, pp. 1959-1962, Nov. 2004.

[24] J. Kim, and S. L. Ariyavistakul, "Optimum 4-transmit-antenna STBC/SFBC with angle feedback and a near-optimum 1-bitfeedback scheme," IEEE Communication Letter, vol. 11, no. 11, pp. 868-870, Nov. 2007.

[25] Zhu Chen, and Moon Ho Lee, "One bit feedback for Quasi-orthogonal space-time block codes based on circulant matrix," IEEE Transactions on wireless communications, vol. 8, no. 7, pp. 3386-3389, Jul. 2009.

[26] Biljana Badic, Markus Rupp, and Hans Weinrichter, "Adaptive channel-matched extended alamouti space-time code exploiting partial feedback,” ETRI Journal, vol. 26, no. 5, pp. 443-451, Oct. 2004.

[27] Yiyue Wu, and Robert Calderbank, "Code diversity in multiple antenna wilreless communication," In: Proc. of International Symp. On Information Technology, Toronto, ON, Canada, pp. 1078-1082, 2008. 
[28] Gerard Favier, Michele N. da Costa, Andre L. F. de Almeida, and Joao Marcos T.Romano, "Tensor space-time (TST) coding for MIMO wireless communication systems," Signal Processing, vol. 92, no. 4, pp. 1079-1092, Mar. 2012.

[29] Walter Freitas, Gerard Favier, and Andre L. F. de Almeida, "Generalized Khatri-Rao and Kronecker space-time coding for MIMO relay systems with closed-form semi-blind receivers,” Signal Processing, vol. 151, pp. 19-31, Oct. 2018.

[30] Chung Buiquang, and Zhongfu Ye, "Constrained ALS-based tensor blind receivers for multi-user MIMO systems," Digital Signal Processing, vol. 84, pp. 69-79, Jan. 2019.

\section{BIOGRAPHIES OF AUTHORS}

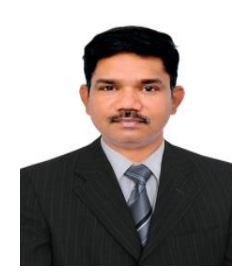

K. Senthil Kumar obtained his Ph.D. at Anna University, Chennai, India in the year 2018. He received his Master Degree from Rajalakshmi Engineering College, Chennai in 2009. In the year 2002, he received his bachelor's degree in Engineering from Bharath Niketan Engineering College, Theni. He is working as an Associate Professor in the department of ECE, Rajalakshmi Engineering College, India. His research interests include wireless communications, information \& coding theory and MIMO wireless communication systems.

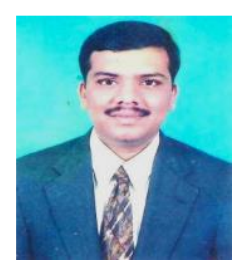

M. Palanivelan is currently working as a Professor \& Head, Department of ECE, Rajalakshmi Engineering College, Chennai. He received his Ph.D at Anna University in the year 2015 and Master Degree from College of Engineering, Guindy, Anna University, in the year 2001. His research interests include Wireless communications, Information and coding theory, Optical Networks and Multiple antenna wireless communication

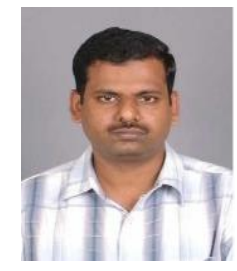

Noor Mohammed Vali Mohamad received his B.E., Degree in Electronics and Communication Engineering from Madras University, Tamil Nadu, India, in 1999 and M.E., Degree in Communication Systems from Madurai Kamarj University, Tamil Nadu, India in 2003. He received his Ph.D in Wireless Communication and Networking from Vellore Institute of Technology (VIT), Vellore, Tamil Nadu, India. Currently he is Associate Professor in School of Electronics Engineering, VIT, Vellore, Tamil Nadu India. He is a member in IEEE and life member in ISTE. He has published more than fifty papers, in reputed journals and conferences. His research interests include Resource allocation in next generation, wireless networks, Massive MIMO and Green Communication.

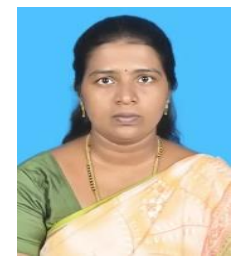

T. Helan Vidhya is a member of ISTE and IACSIT. She completed B.E (Electronics and communication engineering) in Bhajarang Engineering College in 2009 and M.E (Applied Electronics) in St. Joseph's college of Engineering in 2011. Currently, working as an Assistant Professor in the Department of Electronics and Communication Engineering, Rajalakshmi Engineering College, Thandalam and having teaching experience of 8.3 years. Her area of interests are communication engineering and image processing.

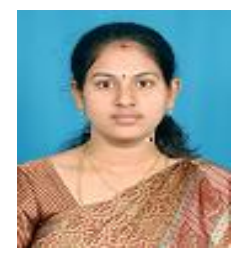

V. Yogalakshmi obtained her Master Degree in Applied Electronics from Arunai Engineering College in the year of 2012. Currently, she is working as an Assistant professor in the Department of Electronics and Communication Engineering, Rajalakshmi Engineering College with 6 years of experience. Her research interest includes wireless communication, speech processing and signal processing.

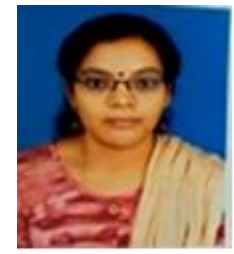

V. Bakyalakshmi obtained her Master Degree in communication systems from Rajalakshmi Engineering College, Chennai in 2016. In the year 2014, she received her bachelor's degree in Electronics and Communication Engineering from Adhiparasakthi College of Engineering, Kalavai. She is working as an assistant professor in the department of ECE, Rajalakshmi Engineering College, India. Her research interests include wireless communication and optical communication. 